\title{
IUFOST2006/1207 \\ Pectin-protein interactions in the cell wall- a biomimetic approach
}

\author{
J. Moffat, A. Macdougall, T. Noel, R. Parker and S. Ring \\ Institute of Food Research, Colney Lane, NR4 7UA Norwich, United Kingdom \\ steve.ring@bbsrc.ac.uk
}

Our previous research examined the polyelectrolyte behaviour of pectin networks and the role of charged residues in influencing the crosslinking and swelling behaviour of the network in water. In addition to inorganic cations, organic polycations such as poly-L-lysine, chitosan, and fragments of the cell wall protein extensin can also crosslink pectin networks. Our current research is examining how these interactions can be used to assemble novel network structures. Using surface plasmon resonance, quartz crystal microblance with dissipation monitoring and fourier transform infrared spectroscopy with attenuated total reflection, we are examining the assembly of pectin networks at surfaces and their interaction with proteins and enzymes including polygalacturonase and pectin methyl esterase. We find that network hydration is sensitive to the action of pectin methyl esterase and this in turn affects its susceptibility to hydrolysis by polygalacturonase. The outcomes of the research will be discussed in the context of both our current understanding of cell wall behaviour and the potential industrial usefulness of these assembled structures. 